J. Nonlinear Var. Anal. 5 (2021), No. 1, pp. 23-42

Available online at http://jnva.biemdas.com

https://doi.org/10.23952/jnva.5.2021.1.03

\title{
ON THE UNIFIED APPROACH BETWEEN UPPER AND LOWER SEMICONTINUOUS DIFFERENTIAL INCLUSIONS
}

\author{
PAOLO CUBIOTTI \\ Department of Mathematical and Computer Sciences, Physical Sciences and Earth Sciences, \\ University of Messina, Messina 98166, Italy
}

\begin{abstract}
In this paper, we consider a $k$-th order differential inclusion with a multifunction $F$ such that its restriction to the complement of a suitable null-measure set is lower semicontinuous and bounded. We prove that there exists an upper semicontinuous multifunction $G$ such that each generalized solution of the corresponding $k$-th order differential inclusion is also a generalized solution of the original differential inclusion. As an application, we prove an existence and qualitative result for the Cauchy problem associated to a class of $k$-th order differential inclusions. In particular, we give sufficient conditions under which the solution multifuncion admits an upper semicontinuous multivalued selection with nonempty compact connected values. Finally, as a further application, we prove an analogous existence and qualitative result for the generalized solutions of the Cauchy problem associated to a class of $k$-th order implicit discontinuous differential equations.
\end{abstract}

Keywords. Differential inclusions; Cauchy problem; Generalized solutions; Differential equations; Discontinuous functions.

\section{INTRODUCTION}

Let $n \in \mathbf{N}, T>0$, and $F:[0, T] \times \mathbf{R}^{n} \rightarrow 2^{\mathbf{R}^{n}}$ be a given multifunction. We recall that a generalized solution of the differential inclusion $u^{\prime} \in F(t, u)$ in $[0, T]$ is an absolutely continuous function $u:[0, T] \rightarrow \mathbf{R}^{n}$ such that

$$
u^{\prime}(t) \in F(t, u(t)) \quad \text { for a.e. } t \in[0, T] .
$$

Such a differential inclusion arises in the study of many problems, including control theory, dynamical systems, discontinuous differential equations and so on. Historically, differential inclusion (1.1) has been studied under two different and separate kind of assumptions, that is,

(i) $F$ is upper semicontinuous with nonempty compact convex values;

(ii) $F$ is lower semicontinuous (or continuous) with nonempty compact (possibly non-convex) values.

As remarked in [1], the results and the techniques for the two classes of differential inclusions are substantially distinct in most literature. In [1], Bressan proved a deep result which allowed to treat the two classes of differential inclusions in a unified way. The following is Bressan's result.

E-mail address: pcubiotti@unime.it.

Received September 17, 2020; Accepted January 10, 2021.

(C)2021 Journal of Nonlinear and Variational Analysis 
Theorem 1.1. [1, the theorem at p.22]. Let $F: \mathbf{R} \times \mathbf{R}^{n} \rightarrow 2^{\mathbf{R}^{n}}$ be a bounded, lower semicontinuous multifunction with nonempty compact values. Then there exists an upper semicontinuous multifunction $G: \mathbf{R} \times \mathbf{R}^{n} \rightarrow 2^{\mathbf{R}^{n}}$, with nonempty compact convex values, such that every generalized solution $u:[\alpha, \beta] \rightarrow \mathbf{R}^{n}$ of the differential inclusion $u^{\prime} \in G(t, u)$ in $[\alpha, \beta]$ (with $[\alpha, \beta]$, real compact interval) is also a solution of $u^{\prime} \in F(t, u)$ in $[\alpha, \beta]$.

Theorem 1.1 was proved by using an existence result for directionally continuous selections ([1, Corollary 2.2]). As a matter of fact, Theorem 1.1 allowed to apply to the lower semicontinuous and nonconvex case many results valid for the upper semicontinuous convex-valued case, obtaining new existence and qualitative results for the generalized solutions of problem 1.1 associated with a lower semicontinuous multifunction $F$ (see, for instance, [1, Theorem 4.2]).

Recently, in [2], Theorem 1.1 was extended to a larger class of multifunctions $F: \mathbf{R} \times \mathbf{R}^{n} \rightarrow$ $2^{\mathbf{R}^{n}}$, which is wider than the one of lower semicontinuous multifunctions. In order to state the main result of [2], we need to introduce some notations. First, we denote by $\mathscr{D}$ the class of all subsets $U \subseteq \mathbf{R} \times \mathbf{R}^{n}$ that can be expressed as a finite union of subsets of $\mathbf{R} \times \mathbf{R}^{n}$, each with at least one projection of null Lebesgue measure. Of course, each set $U \in \mathscr{D}$ has a null $(1+n)$ dimensional Lebesgue measure. Moreover, we denote by $\mathscr{G}_{n}$ the family of all subsets $A \subseteq \mathbf{R}^{n}$ such that, for all $i=1, \ldots, n$, the supremum and the infimum of the projection of $\overline{\operatorname{conv}}(A)$ on the $i$ th axis are both positive or both negative ("드v" stands for the "closed convex hull").

The following is the main result of [2].

Theorem 1.2. [2, Theorem 1.3]. Let $n \in \mathbf{N}$, and let $F: \mathbf{R} \times \mathbf{R}^{n} \rightarrow 2^{\mathbf{R}^{n}}$ be a multifunction. Assume that there exists a set $U \in \mathscr{D}$ such that the multifunction $\left.F\right|_{\left(\mathbf{R} \times \mathbf{R}^{n}\right) \backslash U}$ is bounded and lower semicontinuous with nonempty closed values, and $F\left(\left(\mathbf{R} \times \mathbf{R}^{n}\right) \backslash U\right) \in \mathscr{G}_{n}$.

Then, there exists a multifunction $G: \mathbf{R} \times \mathbf{R}^{n} \rightarrow 2^{\mathbf{R}^{n}}$ such that

(a) $G$ is bounded and upper semicontinuous with nonempty compact convex values.

(b) $G\left(\mathbf{R} \times \mathbf{R}^{n}\right) \subseteq \overline{\operatorname{conv}} F\left(\left(\mathbf{R} \times \mathbf{R}^{n}\right) \backslash U\right)$.

(c) every generalized solution $u:[\alpha, \beta] \rightarrow \mathbf{R}^{n}$ of the differential inclusion $u^{\prime} \in G(t, u)$ in $[\alpha, \beta]$ (with $[\alpha, \beta]$, a real compact interval) is also a solution of the differential inclusion $u^{\prime} \in F(t, u)$ in $[\alpha, \beta]$, and the point $(t, u(t)) \in\left(\mathbf{R} \times \mathbf{R}^{n}\right) \backslash U$ for a.e. $t \in[\alpha, \beta]$.

As a matter of fact, a multifunction $F: \mathbf{R} \times \mathbf{R}^{n} \rightarrow 2^{\mathbf{R}^{n}}$ satisfying the assumptions of Theorem 1.2 can fail to be lower semicontinuous even at all points $(t, x) \in \mathbf{R} \times \mathbf{R}^{n}$ (see [2]). Such a weaker regurarity is paid by assuming that the set $F\left(\left(\mathbf{R} \times \mathbf{R}^{n}\right) \backslash U\right)$ belongs to the family $\mathscr{G}_{n}$. As showed in [2, Example 3.3], Theorem 1.2 does not hold without the latter assumption. As an application of Theorem 1.2, some new existence and qualitative results were obtained for the first-order differential inclusion 1.1 associated with a multifunction $F$, which may not have any semicontinuity property, as well as for first-order implicit differential equations of the type $g\left(u^{\prime}\right)=f(t, u)$ associated with a discontinuous function $f$ (see, e.g., [2, 3]).

Now, fix $n, k \in \mathbf{N}$, let $I \subseteq \mathbf{R}$ be a compact interval, and let $p \in[1,+\infty]$. As usual, we denote by $W^{k, p}\left(I, \mathbf{R}^{n}\right)$ the space of all functions $u \in C^{k-1}\left(I, \mathbf{R}^{n}\right)$ such that $u^{(k-1)}$ is absolutely continuous in $I$ and $u^{(k)} \in L^{p}\left(I, \mathbf{R}^{n}\right)$. Let $T>0$, and let $F:[0, T] \times\left(\mathbf{R}^{n}\right)^{k} \rightarrow 2^{\mathbf{R}^{n}}$ be a given multifunction. We recall that a generalized solution of the differential inclusion

$$
u^{(k)} \in F\left(t, u, u^{\prime}, \ldots, u^{(k-1)}\right) \text { in }[0, T]
$$


is a function $u \in W^{k, 1}\left([0, T], \mathbf{R}^{n}\right)$ such that

$$
u^{(k)}(t) \in F\left(t, u(t), u^{\prime}(t), \ldots, u^{(k-1)}(t)\right) \text { for a.e. } t \in[0, T] .
$$

The aim of this paper is to extend the Theorem 1.2 above to the $k$ th-order differential inclusion 1.3. With respect to the original proof of Theorem 1.2, such an extension is not trivial and requires a more articulate technical construction. The main tool of the proof will be a selection result ([4, Lemma 2.4 ]) for lower semicontinuous multifunctions with possibly nonconvex values. As an application, we shall prove an existence and qualitative result (Theorem 3.2, see below) for the generalized solutions of the Cauchy problem

$$
\begin{cases}u^{(k)} \in F\left(t, u, u^{\prime}, \ldots, u^{(k-1)}\right), & \text { in }[0, T], \\ u^{(i)}(0)=\xi_{i}, & i=0,1, \ldots, k-1,\end{cases}
$$

(where $\xi=\left(\xi_{0}, \xi_{1}, \ldots, \xi_{k-1}\right) \in\left(\mathbf{R}^{n}\right)^{k}$ is a given point) associated with a multifunction $F$, which does not necessarily have any semicontinuity property. In particular, we shall give sufficient conditions under which the multifunction

$$
\xi \in\left(\mathbf{R}^{n}\right)^{k} \rightarrow\left\{u \in W^{k, 1}\left([0, T], \mathbf{R}^{n}\right): u \text { is a generalized solution of (1.4) }\right\}
$$

admits an upper semicontinuous multivalued selection with nonempty compact connected values. We explicitly remark that a multifunction $F$ satisfying the assumption of Theorem 3.2 below can fail to be lower semicontinuous (even separately) even at all points $(t, \xi) \in[0, T] \times$ $\left(\mathbf{R}^{n}\right)^{k}$.

Finally, as a further application, we shall prove an existence and qualitative result (Theorem 4.1, see below) for the generalized solutions of the Cauchy problem

$$
\begin{cases}g\left(u^{(k)}\right)=f\left(t, u, u^{\prime}, \ldots, u^{(k-1)}\right) & \text { in }[0, T], \\ u^{(i)}(0)=\xi_{i}, & i=0,1, \ldots, k-1,\end{cases}
$$

(where $\xi=\left(\xi_{0}, \xi_{1}, \ldots, \xi_{k-1}\right) \in\left(\mathbf{R}^{n}\right)^{k}$ is a given point) associated with a discontinuous function $f$. In particular, we shall give sufficient conditions under which the solution multifunction

$$
\xi \in\left(\mathbf{R}^{n}\right)^{k} \rightarrow \mathscr{S}(\xi):=\left\{u \in W^{k, 1}\left([0, T], \mathbf{R}^{n}\right): u \text { is a generalized solution of (1.5) }\right\}
$$

admits an upper semicontinuous multivalued selection with nonempty compact connected values. As before, our assumptions do not imply the continuity of $f$. As a matter of fact, a function $f:[0, T] \times\left(\mathbf{R}^{n}\right)^{k} \rightarrow \mathbf{R}$ satisfying the assumptions of Theorem 4.1 can be discontinuous, with respect to the second variable, even at all points $\xi \in\left(\mathbf{R}^{n}\right)^{k}$. As regards the function $g$, we only require that it is continuous and locally nonconstant. Counterexamples to possible improvements are also given.

\section{PRELIMinaries}

Let $n, k \in \mathbf{N}$, and let $I \subseteq \mathbf{R}$ be a compact interval. In what follows, we consider the space $W^{k, \infty}\left(I, \mathbf{R}^{n}\right)$ with the initial topology $\sigma_{n, k}^{I}$ that makes the function

$$
u \in W^{k, \infty}\left(I, \mathbf{R}^{n}\right) \rightarrow\left(u, u^{(k)}\right) \in C^{k-1}\left(I, \mathbf{R}^{n}\right) \times L^{\infty}\left(I, \mathbf{R}^{n}\right)
$$

continuous, where the space $C^{k-1}\left(I, \mathbf{R}^{n}\right)$ is considered with its strong topology, and the space $L^{\infty}\left(I, \mathbf{R}^{n}\right)$ is considered with its weak-star topology. 
Let $T>0$, and let $F:[0, T] \times\left(\mathbf{R}^{n}\right)^{k} \rightarrow 2^{\mathbf{R}^{n}}$ be a given multifunction. Let $\xi=\left(\xi_{0}, \xi_{1}, \ldots, \xi_{k-1}\right) \in$ $\left(\mathbf{R}^{n}\right)^{k}$ be fixed. According to Section 1, we recall that a generalized solution of the Cauchy problem (1.4) is a function $u \in W^{k, 1}\left([0, T], \mathbf{R}^{n}\right)$ such that

$$
u^{(k)}(t) \in F\left(t, u(t), u^{\prime}(t), \ldots, u^{(k-1)}(t)\right) \text { for a.e. } t \in[0, T]
$$

and $u^{(i)}(0)=\xi_{i}$ for all $i=0, \ldots, k-1$. Of course, if $F$ is bounded, then every generalized solution of problem (1.4) (as well as of the differential inclusion (1.2) belongs to $W^{k, \infty}\left([0, T], \mathbf{R}^{n}\right)$.

In the following, we shall often make the obvious identification $\left(\mathbf{R}^{n}\right)^{k}=\mathbf{R}^{n k}$. For all $i=$ $0,1, \ldots, n k$, we denote by $P_{j}: \mathbf{R} \times \mathbf{R}^{n k} \rightarrow \mathbf{R}$ the projection over the $j$-th axis. That is, if $(t, x)=$ $\left(t, x_{1}, x_{2}, \ldots, x_{n k}\right) \in \mathbf{R} \times \mathbf{R}^{n k}$, we put

$$
P_{j}(t, x)= \begin{cases}t, & j=0, \\ x_{j}, & j \in\{1,2, \ldots, n k\} .\end{cases}
$$

For each $j \in \mathbf{N}$, we shall denote by $m_{j}$ the $j$-dimensional Lebesgue measure in $\mathbf{R}^{j}$. Moreover, we shall denote by $\mathscr{F}$ the family of all subsets $U \subseteq \mathbf{R} \times \mathbf{R}^{n k}$ such that there exist sets $V_{0}, V_{1}, \ldots, V_{n k} \subseteq \mathbf{R} \times \mathbf{R}^{n k}$, with $m_{1}\left(P_{j}\left(V_{j}\right)\right)=0$ for all $j=0,1 \ldots, n k$, such that $U=\bigcup_{j=0}^{n k} V_{j}$. Of course, any set $U \in \mathscr{F}$ satisfies $m_{n k+1}(U)=0$.

Let $m \in \mathbf{N}$. If $x \in \mathbf{R}^{m}$ and $r>0$, we shall denote by $B_{m}(x, r)$ (resp., $\bar{B}_{m}(x, r)$ ) the open (resp., closed) ball in $\mathbf{R}^{m}$, centered in $x$ with radius $r$, with respect to the Euclidean norm $\|\cdot\|_{m}$ of $\mathbf{R}^{m}$. Finally, we shall denote by $\mathscr{B}(\mathbf{R})$ and $\mathscr{L}([a, b])$, respectively, the Borel family of $\mathbf{R}$ and the family of all Lebesgue measurable subsets of the interval $[a, b]$. For the reader's convenience, we now state some results that will be useful in the sequel. First, we recall the following proposition.

Proposition 2.1. [5, Proposition 2.6]. Let $\psi:[a, b] \times \mathbf{R}^{n} \rightarrow \mathbf{R}^{k}$ be a given function, and let $E \subseteq \mathbf{R}^{n}$ be a Lebesgue measurable set, with $m_{n}(E)=0$. Let $D$ be a countable dense subset of $\mathbf{R}^{n}$, with $D \cap E=\emptyset$. Assume that

(i) for all $t \in[a, b]$, the function $\psi(t, \cdot)$ is bounded;

(ii) for all $x \in D$, the function $\psi(\cdot, x)$ is $\mathscr{L}([a, b])$-measurable.

Let $G:[a, b] \times \mathbf{R}^{n} \rightarrow 2^{\mathbf{R}^{k}}$ be the multifunction defined by setting, for each $(t, x) \in[a, b] \times \mathbf{R}^{n}$,

$$
G(t, x):=\bigcap_{m \in \mathbf{N}} \overline{\operatorname{conv}} \overline{\left(\bigcup_{\substack{y \in D \\\|y-x\|_{n} \leq \frac{1}{m}}}\{\psi(t, y)\}\right)} .
$$

Then,

(a) G has nonempty closed convex values;

(b) for all $x \in \mathbf{R}^{n}$, the multifunction $G(\cdot, x)$ is $\mathscr{L}([a, b])$-measurable;

(c) for all $t \in[a, b]$, the multifunction $G(t, \cdot)$ has closed graph;

(d) if $t \in[a, b]$, and $\left.\psi(t, \cdot)\right|_{\mathbf{R}^{n} \backslash E}$ is continuous at $x \in \mathbf{R}^{n} \backslash E$, then one has

$$
G(t, x)=\{\psi(t, x)\} .
$$

The following result summarizes several results proved in [6, pp.103-109]

Theorem 2.1. Let $x^{*} \in \mathbf{R}^{n}$, and let $\Omega \subseteq \mathbf{R} \times \mathbf{R}^{n}$ be an open set such that $\left(0, x^{*}\right) \in \Omega$. Let $G: \Omega \rightarrow 2^{\mathbf{R}^{n}}$ be an upper semicontinuous multifunction with nonempty compact convex values. 
Assume that there exist $M>0, b>0$ and $T>0$ such that

$$
Q:=[0, T] \times \bar{B}_{n}\left(x^{*}, b+M T\right) \subseteq \Omega \quad \text { and } \quad G(Q) \subseteq \bar{B}_{n}(0, M) .
$$

Then,

(i) for every $\xi \in B_{n}\left(x^{*}, b\right)$, the solution set

$$
\mathscr{T}_{[0, T]}^{G}(\xi):=\left\{u \in W^{1,1}\left([0, T], \mathbf{R}^{n}\right): u(0)=\xi \text { and } u^{\prime}(t) \in G(t, u(t)) \text { a.e. in }[0, T]\right\}
$$

is nonempty. Moreover, the multifunction $\xi \rightarrow \mathscr{T}_{[0, T]}^{G}(\xi)$ is upper semicontinuous from $B_{n}\left(x^{*}, b\right)$ to $W^{1, \infty}\left([0, T], \mathbf{R}^{n}\right)$, with nonempty, compact and connected values;

(ii) the multifunction $\xi \rightarrow \mathscr{A}_{[0, T]}^{G}(\xi):=\left\{u(T): u \in \mathscr{T}_{[0, T]}^{G}(\xi)\right\}$ is upper semicontinuous from $B_{n}\left(x^{*}, b\right)$ to $\mathbf{R}^{n}$ with nonempty compact connected values.

Finally, we prove the following fact, which will be crucial in the sequel.

Proposition 2.2. Let $n, k \in \mathbf{N}$, and let $I=[0, a]$ be a compact interval. For each function $v \in W^{k, \infty}\left(I, \mathbf{R}^{n}\right)$, let

$$
y_{v}: I \rightarrow\left(\mathbf{R}^{n}\right)^{k}
$$

be defined by putting, for every $s \in I$,

$$
y_{v}(s)=\left(v(s), v^{\prime}(s), \ldots, v^{(k-1)}(s)\right) .
$$

Let $E:=\left\{y_{v}: v \in W^{k, \infty}\left(I, \mathbf{R}^{n}\right)\right\}$, and let

$$
P_{1}^{*}:\left[W^{1, \infty}\left(I, \mathbf{R}^{n}\right)\right]^{k} \rightarrow W^{1, \infty}\left(I, \mathbf{R}^{n}\right)
$$

be the first projection. Then, $E$ is a closed subset of $\left[W^{1, \infty}\left(I, \mathbf{R}^{n}\right)\right]^{k}$ and the function

$$
\left.P_{1}^{*}\right|_{E}:\left(E,\left(\sigma_{n, 1}^{I}\right)^{k}\right) \rightarrow\left(W^{k, \infty}\left(I, \mathbf{R}^{n}\right), \sigma_{n, k}^{I}\right)
$$

is surjective and continuous.

Proof. Clearly, one has $y_{v} \in\left[W^{1, \infty}\left(I, \mathbf{R}^{n}\right)\right]^{k}$ for every $v \in W^{k, \infty}\left(I, \mathbf{R}^{n}\right)$. In order to check that $E$ is a closed subset of $\left[W^{1, \infty}\left(I, \mathbf{R}^{n}\right)\right]^{k}$, let $\left\{v_{\alpha}\right\}_{\alpha \in \Lambda}$ be a net in $W^{k, \infty}\left(I, \mathbf{R}^{n}\right)$ and let

$$
w=\left(w_{0}, w_{1}, \ldots, w_{k-1}\right) \in\left[W^{1, \infty}\left(I, \mathbf{R}^{n}\right)\right]^{k},
$$

such that $\left\{y_{v_{\alpha}}\right\}_{\alpha \in \Lambda} \rightarrow w$ in $\left[W^{1, \infty}\left(I, \mathbf{R}^{n}\right)\right]^{k}$ with respect to the topology $\left(\sigma_{n, 1}^{I}\right)^{k}$. Therefore, for every $j=0, \ldots, k-1$, we have $\left\{v_{\alpha}^{(j)}\right\}_{\alpha \in \Lambda} \rightarrow w_{j}$ in $W^{1, \infty}\left(I, \mathbf{R}^{n}\right)$ with respect to the topology $\sigma_{n, 1}^{I}$. By the definition of the topology $\sigma_{n, 1}^{I}$, this means that

$$
\left\{\left(v_{\alpha}^{(j)}, v_{\alpha}^{(j+1)}\right)\right\}_{\alpha \in \mathfrak{E}} \rightarrow\left(w_{j}, w_{j}^{\prime}\right) \quad \text { in } \quad C^{0}\left(I, \mathbf{R}^{n}\right) \times L^{\infty}\left(I, \mathbf{R}^{n}\right)
$$

for every $j=0, \ldots, k-1$, where $C^{0}\left(I, \mathbf{R}^{n}\right)$ is considered with its strong topology, and the space $L^{\infty}\left(I, \mathbf{R}^{n}\right)$ with its weak-star topology. Therefore, for every $j=0, \ldots, k-1$, one has that

$$
\left\{v_{\alpha}^{(j)}\right\}_{\alpha \in \Lambda} \rightarrow w_{j} \quad \text { strongly in } C^{0}\left(I, \mathbf{R}^{n}\right)
$$

and

$$
\left\{v_{\alpha}^{(j+1)}\right\}_{\alpha \in \Lambda} \rightarrow w_{j}^{\prime} \quad \text { weakly-star in } L^{\infty}\left(I, \mathbf{R}^{n}\right) .
$$


In particular, for every $i=1, \ldots, k-1$, one has at same time that $\left\{v_{\alpha}^{(i)}\right\}_{\alpha \in \Lambda} \rightarrow w_{i}$ strongly in $C^{0}\left(I, \mathbf{R}^{n}\right)$ and $\left\{v_{\alpha}^{(i)}\right\}_{\alpha \in \Lambda} \rightarrow w_{i-1}^{\prime}$ weakly-star in $L^{\infty}\left(I, \mathbf{R}^{n}\right)$. Consequently, we get that, for every $i=1, \ldots, k-1$,

$$
w_{i}(s)=w_{i-1}^{\prime}(s) \text { for a.e. } s \in I .
$$

Now, fix $i \in\{1, \ldots, k-1\}$. From the absolute continuity of $w_{i-1}$ and (2.1), we have

$$
w_{i-1}(s)=w_{i-1}(0)+\int_{0}^{s} w_{i-1}^{\prime}(z) d z=w_{i-1}(0)+\int_{0}^{s} w_{i}(z) d z
$$

for all $s \in I$. Since $w_{i}$ is continuous, we get that $w_{i-1} \in C^{1}\left(I, \mathbf{R}^{n}\right)$ and

$$
w_{i-1}^{\prime}(s)=w_{i}(s) \text { for all } s \in I .
$$

Therefore, we have proved that, for every $i=1, \ldots, k-1, w_{i-1} \in C^{1}\left(I, \mathbf{R}^{n}\right)$ and $w_{i-1}^{\prime}=w_{i}$. This easily implies that $w_{0} \in W^{k, \infty}\left(I, \mathbf{R}^{n}\right)$ and $w=y_{w_{0}}$. Hence, $w \in E$ and $E$ is closed, as claimed.

Of course, $P_{1}^{*}(E)=W^{k, \infty}\left(I, \mathbf{R}^{n}\right)$. We now prove that

$$
\left.P_{1}^{*}\right|_{E}:\left(E,\left(\sigma_{n, 1}^{I}\right)^{k}\right) \rightarrow\left(W^{k, \infty}\left(I, \mathbf{R}^{n}\right), \sigma_{n, k}^{I}\right)
$$

is continuous. To this aim, let $\left(\bar{w}_{\alpha}\right)_{\alpha \in \Lambda^{\prime}}$ be a net in $E$, converging to a point $\bar{w} \in E$ with respect to the topology $\left(\sigma_{n, 1}^{I}\right)^{k}$. For each $\alpha \in \Lambda^{\prime}$, let $\bar{v}_{\alpha} \in W^{k, \infty}\left(I, \mathbf{R}^{n}\right)$ be such that $\bar{w}_{\alpha}=y_{\bar{v}_{\alpha}}$. Moreover, let $\bar{v} \in W^{k, \infty}\left(I, \mathbf{R}^{n}\right)$ be such that $\bar{w}=y_{\bar{v}}$. Hence, we have that

$$
\left\{\left(\bar{v}_{\alpha}, \bar{v}_{\alpha}^{\prime}, \ldots, \bar{v}_{\alpha}^{(k-1)}\right)\right)_{\alpha \in \Lambda^{\prime}} \rightarrow\left(\bar{v}, \bar{v}^{\prime}, \ldots, \bar{v}^{(k-1)}\right)
$$

with respect to the topology $\left(\sigma_{n, 1}^{I}\right)^{k}$. Consequently, we have

$$
\left\{\bar{v}_{\alpha}^{(j)}\right\}_{\alpha \in \Lambda^{\prime}} \rightarrow \bar{v}^{(j)} \quad \text { for every } \quad j=0, \ldots, k-1,
$$

with respect to the topology $\sigma_{n, 1}^{I}$. By the definition of the topology $\sigma_{n, 1}^{I}$, this means that

$$
\left\{\left(\bar{v}_{\alpha}^{(j)}, \bar{v}_{\alpha}^{(j+1)}\right)\right\}_{\alpha \in \Lambda^{\prime}} \rightarrow\left(\bar{v}^{(j)}, \bar{v}^{(j+1)}\right) \quad \text { for every } \quad j=0, \ldots, k-1
$$

in $C^{0}\left(I, \mathbf{R}^{n}\right) \times \Lambda^{\infty}\left(I, \mathbf{R}^{n}\right)$ (where $C^{0}\left(I, \mathbf{R}^{n}\right)$ is considered with its strong topology, and $L^{\infty}\left(I, \mathbf{R}^{n}\right)$ is considered with its weak-star topology). Hence, we have that $\left\{\bar{v}_{\alpha}^{(j)}\right\}_{\alpha \in \Lambda^{\prime}} \rightarrow \bar{v}^{(j)}$ for every $j=0, \ldots, k-1$ with respect to the strong topology of $C^{0}\left(I, \mathbf{R}^{n}\right)$, and $\left\{\bar{v}_{\alpha}^{(k)}\right\}_{\alpha \in \Lambda^{\prime}} \rightarrow \bar{v}^{(k)}$ with respect to the weak-star topology of $L^{\infty}\left(I, \mathbf{R}^{n}\right)$. Therefore, $\left\{\bar{v}_{\alpha}\right\}_{\alpha \in \Lambda^{\prime}} \rightarrow \bar{v}$ with respect to the strong topology of $C^{k-1}\left(I, \mathbf{R}^{n}\right)$, and thus

$$
\left\{\left(\bar{v}_{\alpha}, \bar{v}_{\alpha}^{(k)}\right)\right\}_{\alpha \in \Lambda^{\prime}} \rightarrow\left(\bar{v}, \bar{v}^{(k)}\right)
$$

in $C^{k-1}\left(I, \mathbf{R}^{n}\right) \times L^{\infty}\left(I, \mathbf{R}^{n}\right)$, where the first space is taken with its strong topology, and the second space with its weak-star topology. Therefore, we have that $\left\{\bar{v}_{\alpha}\right\}_{\alpha \in \Lambda^{\prime}} \rightarrow \bar{v}$ with respect to the topology $\sigma_{n, k}^{I}$. This implies that $\left\{P_{1}^{*}\left(\bar{w}_{\alpha}\right)\right\}_{\alpha \in \Lambda^{\prime}} \rightarrow P_{1}^{*}(\bar{w})$ with respect to the topology $\sigma_{n, k}^{I}$. Thus, our claim follows. The proof is complete.

For the basic facts and definitions about multifunctions, we refer the reader to [7, 8]. 


\section{MAIN RESULTS}

The following is our main result.

Theorem 3.1. Let $n, k \in \mathbf{N}$, and $T>0$. Let $F:[0, T] \times\left(\mathbf{R}^{n}\right)^{k} \rightarrow 2^{\mathbf{R}^{n}}$ be a given multifunction. Put $S:=[0, T] \times\left(\mathbf{R}^{n}\right)^{k}$. Assume that there exists a set $U \subseteq S$, with $U \in \mathscr{F}$ such that

(i) $\left.F\right|_{S \backslash U}$ is lower semicontinuous with nonempty and closed values;

(ii) $F(S \backslash U) \in \mathscr{G}_{n}$ and $F(S \backslash U)$ is bounded.

Then, there exists a bounded and upper semicontinuous multifunction $G: \mathbf{R} \times\left(\mathbf{R}^{n}\right)^{k} \rightarrow 2^{\mathbf{R}^{n}}$ with nonempty convex and compact values such that

(a) $G\left(\mathbf{R} \times\left(\mathbf{R}^{n}\right)^{k}\right) \subseteq \overline{\operatorname{conv}}(F(S \backslash U))$;

(b) every generalized solution $u \in W^{k, \infty}\left([0, T], \mathbf{R}^{n}\right)$ of the inclusion

$$
u^{(k)} \in G\left(t, u, u^{\prime}, \ldots, u^{(k-1)}\right)
$$

in $[0, T]$ is also a generalized solution of the inclusion $u^{(k)} \in F\left(t, u, u^{\prime}, \ldots, u^{(k-1)}\right)$ in $[0, T]$.

(c) for every generalized solution $u \in W^{k, \infty}\left([0, T], \mathbf{R}^{n}\right)$ of the inclusion

$$
u^{(k)} \in G\left(t, u, u^{\prime}, \ldots, u^{(k-1)}\right)
$$

in $[0, T]$, one has

$$
\left(t, u(t), u^{\prime}(t), \ldots, u^{(k-1)}(t)\right) \in S \backslash U \quad \text { for a.e. } \quad t \in[0, T] .
$$

Proof. Let $r>0$ be such that $F(S \backslash U) \subseteq \bar{B}_{n}(0, r)$. By the definition of the family $\mathscr{F}$, there exists $W_{0}, W_{1}, \ldots, W_{n k} \subseteq \mathbf{R} \times \mathbf{R}^{n k}$, with $m_{1}\left(P_{j}\left(W_{j}\right)\right)=0$ for all $j=0,1 \ldots, n k$, such that $U=\bigcup_{i=0}^{n k} W_{j}$. Let $H_{0}, H \in \mathscr{B}(\mathbf{R})$, with $H_{0} \subseteq[0, T]$ and $m_{1}\left(H_{0}\right)=m_{1}(H)=0$, be such that

$$
P_{0}\left(W_{0}\right) \subseteq H_{0} \quad \text { and } \quad \bigcup_{j=1}^{n k} P_{j}\left(W_{j}\right) \subseteq H .
$$

Put

$$
V^{*}:=\left([0, T] \backslash H_{0}\right) \times(\mathbf{R} \backslash H)^{n k}
$$

Obvious, we have

$$
V^{*} \subseteq S \backslash U
$$

By [4, Lemma 2.4], there exist two sets $H_{0}^{\prime}, H^{\prime} \in \mathscr{B}(\mathbf{R})$ with $H_{0}^{\prime} \subseteq[0, T]$ and $m_{1}\left(H_{0}^{\prime}\right)=m_{1}\left(H^{\prime}\right)=$ 0 , and a function $f: V^{*} \rightarrow \mathbf{R}^{n}$ such that

(i) $)^{\prime} f(t, \xi) \in F(t, \xi)$ for all $(t, \xi) \in V^{*}$;

(ii) ${ }^{\prime} f$ is continuous at every point

$$
(t, \xi) \in\left([0, T] \backslash\left(H_{0} \cup H_{0}^{\prime}\right)\right) \times\left(\mathbf{R} \backslash\left(H \cup H^{\prime}\right)\right)^{n k} .
$$

Fix a point $z^{*} \in f\left(V^{*}\right)$, and let $f^{*}: \mathbf{R} \times \mathbf{R}^{n k} \rightarrow \mathbf{R}^{n}$ be defined by putting

$$
f^{*}(t, \xi)= \begin{cases}f(t, \xi), & \text { if }(t, \xi) \in V^{*} \\ z^{*} & \text { if }(t, \xi) \in\left(\mathbf{R} \times \mathbf{R}^{n k}\right) \backslash V^{*}\end{cases}
$$

Let

$$
Z:=\left[\left(H_{0} \cup H_{0}^{\prime} \cup\{0, T\}\right) \times \mathbf{R}^{n k}\right] \cup\left[S \cap \bigcup_{i=1}^{n k} P_{i}^{-1}\left(H \cup H^{\prime}\right)\right]
$$


Of course, $Z \subseteq S$. We claim that $\left.f^{*}\right|_{\left(\mathbf{R} \times \mathbf{R}^{n k}\right) \backslash Z}$ is continuous. To see this, fix $(t, \xi) \in\left(\mathbf{R} \times \mathbf{R}^{n k}\right) \backslash$ $Z$.

First, we assume that $(t, \xi) \in S$. Hence,

$$
(t, \xi) \in S \backslash Z=(] 0, T\left[\times \mathbf{R}^{n k}\right) \backslash Z .
$$

Since $(S \backslash Z) \subseteq V^{*}$, we get

$$
\left.f^{*}\right|_{(] 0, T\left[\times \mathbf{R}^{n k}\right) \backslash Z}=\left.f\right|_{(] 0, T\left[\times \mathbf{R}^{n k}\right) \backslash Z} \cdot
$$

By (ii) $)^{\prime}$, the function $\left.f\right|_{\left(j 0, T\left[\times \mathbf{R}^{n k}\right) \backslash Z\right.}$ is continuous. Consequently, by (3.2), we have that $\left.f^{*}\right|_{(] 0, T\left[\times \mathbf{R}^{n k}\right) \backslash Z}$ is continuous. Since (] $0, T\left[\times \mathbf{R}^{n k}\right) \backslash Z$ is an open neighborhood of $(t, \xi)$ in $\left(\mathbf{R} \times \mathbf{R}^{n k}\right) \backslash Z$, we have that $\left.f^{*}\right|_{\left(\mathbf{R} \times \mathbf{R}^{n k}\right) \backslash Z}$ is continuous at $(t, \xi)$, as desired.

Conversely, we assume that $(t, \xi) \notin S$. Since $\left(\mathbf{R} \times \mathbf{R}^{n k}\right) \backslash S$ is open in $\mathbf{R} \times \mathbf{R}^{n k}$ and

$$
\left(\mathbf{R} \times \mathbf{R}^{n k}\right) \backslash S \subseteq\left(\mathbf{R} \times \mathbf{R}^{n k}\right) \backslash Z,
$$

the set $\left(\mathbf{R} \times \mathbf{R}^{n k}\right) \backslash S$ is open in $\left(\mathbf{R} \times \mathbf{R}^{n k}\right) \backslash Z$. Since $f^{*}$ is constant over $\left(\mathbf{R} \times \mathbf{R}^{n k}\right) \backslash S$, we get that $\left.f^{*}\right|_{\left(\mathbf{R} \times \mathbf{R}^{n k}\right) \backslash Z}$ is continuous at $(t, \xi)$, as desired.

Now, taking into account (3.1) and (i) ${ }^{\prime}$, we have

$$
f^{*}\left(\mathbf{R} \times \mathbf{R}^{n k}\right)=f\left(V^{*}\right) \subseteq F\left(V^{*}\right) \subseteq F(S \backslash U) \subseteq \bar{B}_{n}(0, r) .
$$

In particular, by assumption (ii), we get that $f^{*}\left(\mathbf{R} \times \mathbf{R}^{n k}\right) \in \mathscr{G}_{n}$.

Let $D \subseteq\left(\mathbf{R} \times \mathbf{R}^{n k}\right) \backslash Z$ be a countable set, which is dense in $\mathbf{R} \times \mathbf{R}^{n k}$. Of course, such a set $D$ exists due to $m_{1+n k}(Z)=0$.

Now, let $G: \mathbf{R} \times \mathbf{R}^{n k} \rightarrow 2^{\mathbf{R}^{n}}$ be the multifunction defined by setting, for each $(t, \xi) \in \mathbf{R} \times \mathbf{R}^{n k}$,

$$
G(t, \xi):=\bigcap_{m \in \mathbf{N}} \overline{\operatorname{conv}} \overline{\left(\bigcup_{\substack{(l, \eta) \in D \\\|(l, \eta)-(t, \xi)\|_{1+n k} \leq \frac{1}{m}}}\left\{f^{*}(l, \eta)\right\}\right)}
$$

Using Proposition 2.1, and taking into account that $\left.f^{*}\right|_{\left(\mathbf{R} \times \mathbf{R}^{n k}\right) \backslash Z}$ is continuous, we get that

(i) ${ }^{\prime \prime}$ the multifunction $G$ has closed graph and nonempty closed convex values;

(ii) ${ }^{\prime \prime}$ for every $(t, \xi) \in\left(\mathbf{R} \times \mathbf{R}^{n k}\right) \backslash Z$, one has

$$
G(t, \xi)=\left\{f^{*}(t, \xi)\right\} .
$$

In particular, since $(S \backslash Z) \subseteq V^{*}$, we have that $\left.f^{*}\right|_{S \backslash Z}=\left.f\right|_{S \backslash Z \text {. Hence }}$

$$
G(t, \xi)=\{f(t, \xi)\} \quad \text { for all } \quad(t, \xi) \in S \backslash Z .
$$

Moreover, from the above construction, we get

$$
G\left(\mathbf{R} \times \mathbf{R}^{n k}\right) \subseteq \overline{\operatorname{conv}}\left(F\left(V^{*}\right)\right) \subseteq \overline{\operatorname{conv}}(F(S \backslash U)),
$$

which implies that

$$
G\left(\mathbf{R} \times \mathbf{R}^{n k}\right) \in \mathscr{G}_{n} \quad \text { and } \quad G\left(\mathbf{R} \times \mathbf{R}^{n k}\right) \subseteq \bar{B}_{n}(0, r) .
$$

From (3.5), (i)" and [8, Theorem 7.1.16], we have that $G$ is upper semicontinuous. Of course, we can regard the multifunction $G$ as defined on $\mathbf{R} \times\left(\mathbf{R}^{n}\right)^{k}$ by means of the identification $\left(\mathbf{R}^{n}\right)^{k}=\mathbf{R}^{n k}$. Now, let $u \in W^{k, \infty}\left([0, T], \mathbf{R}^{n}\right)$ be a solution of the differential inclusion

$$
u^{(k)} \in G\left(t, u, u^{\prime}, \ldots, u^{(k-1)}\right) \quad \text { in }[0, T] .
$$


Hence, there exists $\S_{0} \in \mathscr{L}([0, T])$, with $m_{1}\left(\S_{0}\right)=0$ such that

$$
u^{(k)}(t) \in G\left(t, u(t), u^{\prime}(t), \ldots, u^{(k-1)}(t)\right) \quad \text { for all } t \in[0, T] \backslash \S_{0} .
$$

In particular, by (3.4), we have

$$
u^{(k)}(t) \in \overline{\operatorname{conv}}\left(F\left(V^{*}\right)\right) \subseteq \overline{\operatorname{conv}}(F(S \backslash U)) \text { for all } t \in[0, T] \backslash \S_{0} .
$$

Fix $i \in\{1, \ldots, n\}$, and let us denote by $u_{i}$ the $i$-th component of the function $u$. From (3.5) and (3.6), we get that $u_{i}^{(k)}(t)$ has constant sign for all $t \in[0, T] \backslash \S_{0}$. Assume that

$$
u_{i}^{(k)}(t)>0 \text { for all } t \in[0, T] \backslash \S_{0}
$$

(if $u_{i}^{(k)}(t)<0$ for all $t \in[0, T] \backslash \S_{0}$, then the argument is analogous). This implies that the absolutely continuous function $u_{i}^{(k-1)}$ is strictly increasing in $[0, T]$ (with a.e. positive derivative). Applying [9, Theorem 2], we have that $\left(u_{i}^{(k-1)}\right)^{-1}$ is absolutely continuous. Hence, it follows from [10, Theorem 18.25] that

$$
C_{i, k-1}:=\left(u_{i}^{(k-1)}\right)^{-1}\left(H \cup H^{\prime}\right)=\left\{t \in[0, T]: u_{i}^{(k-1)}(t) \in H \cup H^{\prime}\right\}
$$

has null Lebesgue measure. Since $u_{i}^{(k-1)}$ is strictly increasing in $[0, T]$, there exists a partition

$$
0=t_{k-1,0}<\ldots<t_{k-1, j_{k-1}}=T
$$

(with $j_{k-1} \leq 2$ ) of the interval $[0, T]$ such that $u_{i}^{(k-1)}$ has constant sign over each interval ]$t_{k-1, l-1}, t_{k-1, l}\left[\right.$ (in particular, $u_{i}^{(k-1)}(t) \neq 0$ on each interval $] t_{k-1, l-1}, t_{k-1, l}[$ ). This implies that, for every $l=1, \ldots, j_{k-1}$, the function $\left.u_{i}^{(k-2)}\right|_{\left[t_{k-1, l-1}, t_{k-1, l}\right]}$ is strictly monotone. Then, for each $l=1, \ldots, j_{k-1},[9$, Theorem 2] yields that the function

$$
\left(\left.u_{i}^{(k-2)}\right|_{\left[t_{k-1, l-1}, t_{k-1, l}\right]}\right)^{-1}
$$

is absolutely continuous. So, in view of [10, Theorem 18.25], it maps null sets into null sets. Consequently, for every $l=1, \ldots, j_{k-1}$, the set

$$
\left(\left.u_{i}^{(k-2)}\right|_{\left[t_{k-1, l-1}, t_{k-1, l}\right]}\right)^{-1}\left(H \cup H^{\prime}\right)=\left\{t \in\left[t_{k-1, l-1}, t_{k-1, l}\right]: u_{i}^{(k-2)}(t) \in H \cup H^{\prime}\right\}
$$

has null Lebesgue measure. Thus, it is easily seen that the set

$$
C_{i, k-2}:=\left(u_{i}^{(k-2)}\right)^{-1}\left(H \cup H^{\prime}\right)=\left\{t \in[0, T]: u_{i}^{(k-2)}(t) \in H \cup H^{\prime}\right\}
$$

has null Lebesgue measure. Since the function $u_{i}^{(k-2)}$ is strictly monotone on each interval $\left[t_{k-1, l-1}, t_{k-1, l}\right]$ with $l=1, \ldots, j_{k-1}$, we have that there exists a partition

$$
0=t_{k-2,0}<\ldots t_{k-2, j_{k-2}}=T
$$

(with $j_{k-2} \leq 4$ ) of the interval $[0, T]$ such that $u_{i}^{(k-2)}$ has constant sign over each interval ]$t_{k-2, l-1}, t_{k-2, l}\left[\right.$ (in particular, $u_{i}^{(k-2)}(t) \neq 0$ on each interval $] t_{k-2, l-1}, t_{k-2, l}[$ ). This implies that, for every $l=1, \ldots, j_{k-2},\left.u_{i}^{(k-3)}\right|_{\left[t_{k-2, l-1}, t_{k-2, l}\right]}$ is strictly monotone. Then, by [9, Theorem 2], for each $l=1, \ldots, j_{k-2}$, the function

$$
\left(\left.u_{i}^{(k-3)}\right|_{\left[t_{k-2, l-1}, t_{k-2, l}\right]}\right)^{-1}
$$


is absolutely continuous. Consequently, it follows from [10, Theorem 18.25] that the set

$$
C_{i, k-3}:=\left[u_{i}^{(k-3)}\right]^{-1}\left(H \cup H^{\prime}\right)=\left\{t \in[0, T]: u_{i}^{(k-3)}(t) \in H \cup H^{\prime}\right\}
$$

has null Lebesgue measure. If we apply recursively the same argument, we get that, for every $j=0, \ldots, k-1$, the set

$$
C_{i, j}:=\left[u_{i}^{(j)}\right]^{-1}\left(H \cup H^{\prime}\right)=\left\{t \in[0, T]: u_{i}^{(j)}(t) \in H \cup H^{\prime}\right\},
$$

has null Lebesgue measure. At this point, put

$$
C:=\{0, T\} \cup H_{0} \cup H_{0}^{\prime} \cup \S_{0} \cup\left[\bigcup_{\substack{i=1, \ldots, n \\ j=0, \ldots, k-1}} C_{i, j}\right] .
$$

The above construction implies that $m_{1}(C)=0$. Choose a point $\left.t \in[0, T] \backslash C=\right] 0, T[\backslash C$. From the definition of the sets $C_{i, j}$, we have that

$$
u_{i}^{(j)}(t) \notin H \cup H^{\prime}
$$

for every $i=1, \ldots, n$ and every $j=0, \ldots, k-1$. Hence

$$
\left(t, u(t), u^{\prime}(t), \ldots, u^{(k-1)}(t)\right) \in S \backslash Z .
$$

By (3.3), we then get

$$
G\left(t, u(t), u^{\prime}(t), \ldots, u^{(k-1)}(t)\right)=\left\{f\left(t, u(t), u^{\prime}(t), \ldots, u^{(k-1)}(t)\right)\right\} .
$$

Taking into account (i) ${ }^{\prime}$ and (3.6), we then have

$$
u^{(k)}(t)=f\left(t, u(t), u^{\prime}(t), \ldots, u^{(k-1)}(t)\right) \in F\left(t, u(t), u^{\prime}(t), \ldots, u^{(k-1)}(t)\right) .
$$

Consequently, since $m_{1}(C)=0$, the function $u$ is a generalized solution in $[0, T]$ of the differential inclusion

$$
u^{(k)} \in F\left(t, u, u^{\prime}, \ldots, u^{(k-1)}\right) .
$$

Since $(S \backslash Z) \subseteq V^{*} \subseteq(S \backslash U)$, the relation (3.7) implies that

$$
\left(t, u(t), u^{\prime}(t), \ldots, u^{(k-1)}(t)\right) \in S \backslash U \quad \text { for a.e. } t \in[0, T] .
$$

The proof is now complete.

As an application of Theorem 3.1, we now prove the following existence and qualitative result for higher-order differential inclusions.

Theorem 3.2. Let $n, k \in \mathbf{N}$ and $T>0$, and let $F:[0, T] \times\left(\mathbf{R}^{n}\right)^{k} \rightarrow 2^{\mathbf{R}^{n}}$ be a given multifunction. Put $S:=[0, T] \times\left(\mathbf{R}^{n}\right)^{k}$. Assume that there exists a set $U \subseteq S$ with $U \in \mathscr{F}$ such that

(i) $\left.F\right|_{S \backslash U}$ is lower semicontinuous with nonempty and closed values;

(ii) $F(S \backslash U) \in \mathscr{G}_{n}$ and $F(S \backslash U)$ is bounded.

Then, for every $\xi=\left(\xi_{0}, \xi_{1}, \ldots, \xi_{k-1}\right) \in\left(\mathbf{R}^{n}\right)^{k}$, the solution set

$$
\mathscr{T}_{[0, T]}^{F}(\xi):=\left\{u \in W^{k, 1}\left([0, T], \mathbf{R}^{n}\right): u \text { is a generalized solution of (1.4) }\right\}
$$

of problem (1.4) is nonempty. Moreover, there exists a multifunction

$$
\Phi:\left(\mathbf{R}^{n}\right)^{k} \rightarrow 2^{W^{k, \infty}\left([0, T] ; \mathbf{R}^{n}\right)}
$$

such that

(a) $\Phi(\xi) \subseteq \mathscr{T}_{[0, T]}^{F}(\xi)$ for all $\xi \in\left(\mathbf{R}^{n}\right)^{k}$ 
(b) $\Phi$ is upper semicontinuous (with respect to the topology $\sigma_{n, k}^{[0, T]}$ of $W^{k, \infty}\left([0, T] ; \mathbf{R}^{n}\right)$ ) with nonempty, compact and connected values;

(c) the multifunction

$$
\xi \in\left(\mathbf{R}^{n}\right)^{k} \rightarrow\{u(T): u \in \Phi(\xi)\}
$$

is upper semicontinuous with nonempty connected and compact values;

(d) for every $\xi \in\left(\mathbf{R}^{n}\right)^{k}$ and every $u \in \Phi(\xi)$, one has

$$
\left(t, u(t), u^{\prime}(t), \ldots, u^{(k-1)}(t)\right) \in S \backslash U \quad \text { for a.e. } \quad t \in[0, T]
$$

(e) the multifunction

$$
\xi \in\left(\mathbf{R}^{n}\right)^{k} \rightarrow\left\{u^{(k)} \in L^{\infty}\left([0, T], \mathbf{R}^{n}\right): u \in \Phi(\xi)\right\}
$$

is upper semicontinuous (with compact connected values) with respect to the weak-star topology of $L^{\infty}\left([0, T], \mathbf{R}^{n}\right)$.

Proof. Let $r>0$ such that $F(S \backslash U) \subseteq \bar{B}_{n}(0, r)$. By the definition of the family $\mathscr{F}$, there exists sets $W_{0}, W_{1}, \ldots, W_{n k} \subseteq \mathbf{R} \times \mathbf{R}^{n k}$, with $m_{1}\left(P_{j}\left(W_{j}\right)\right)=0$ for all $j=0,1 \ldots, n k$, such that $U=$ $\bigcup_{i=0}^{n k} W_{j}$. Let $H_{0}, H \in \mathscr{B}(\mathbf{R})$, with $H_{0} \subseteq[0, T]$ and $m_{1}\left(H_{0}\right)=m_{1}(H)=0$, be such that

$$
P_{0}\left(W_{0}\right) \subseteq H_{0} \quad \text { and } \quad \bigcup_{j=1}^{n k} P_{j}\left(W_{j}\right) \subseteq H .
$$

Of course, we have that

$$
\Omega:=\left([0, T] \backslash H_{0}\right) \times(\mathbf{R} \backslash H)^{n k} \subseteq S \backslash U .
$$

By the definition of the family $\mathscr{G}_{n}$ and assumptions (i) and (ii), we have that $\left.F\right|_{\Omega}$ is lower semicontinuous with nonempty and closed values, $F(\Omega) \in \mathscr{G}_{n}$ and $F(\Omega) \subseteq \bar{B}_{n}(0, r)$.

Now, let $I:=[0,1 / 2]$, and let $F^{*}: I \times\left(\mathbf{R}^{n}\right)^{k} \rightarrow 2^{\mathbf{R}^{n}}$ be defined by putting, for each $\left(s, \xi_{0}, \xi_{1}, \ldots\right.$, $\left.\xi_{k-1}\right) \in I \times\left(\mathbf{R}^{n}\right)^{k}$

$$
F^{*}\left(s, \xi_{0}, \xi_{1}, \ldots, \xi_{k-1}\right)=2^{k} T^{k} F\left(2 T s, \xi_{0}, \frac{1}{2 T} \xi_{1}, \frac{1}{2^{2} T^{2}} \xi_{2}, \ldots, \frac{1}{2^{k-1} T^{k-1}} \xi_{k-1}\right) .
$$

Consider the function $f: I \times\left(\mathbf{R}^{n}\right)^{k} \rightarrow[0, T] \times\left(\mathbf{R}^{n}\right)^{k}$ defined by putting, for each $\left(s, \xi_{0}, \xi_{1}, \ldots\right.$, $\left.\xi_{k-1}\right) \in I \times\left(\mathbf{R}^{n}\right)^{k}$

$$
f\left(s, \xi_{0}, \xi_{1}, \ldots, \xi_{k-1}\right)=\left(2 T s, \xi_{0}, \frac{1}{2 T} \xi_{1}, \frac{1}{2^{2} T^{2}} \xi_{2}, \ldots, \frac{1}{2^{k-1} T^{k-1}} \xi_{k-1}\right) .
$$

It is immediate to check that $f$ is continuous. Moreover, one has

$$
F^{*}\left(s, \xi_{0}, \xi_{1}, \ldots, \xi_{k-1}\right)=2^{k} T^{k} F\left(f\left(s, \xi_{0}, \xi_{1}, \ldots, \xi_{k-1}\right)\right)
$$

for every $\left(s, \xi_{0}, \xi_{1}, \ldots, \xi_{k-1}\right) \in I \times\left(\mathbf{R}^{n}\right)^{k}$. Now, put

$$
H_{0}^{*}:=\frac{1}{2 T} H_{0}, \quad H^{*}:=\bigcup_{i=0}^{k-1}\left(2^{i} T^{i} H\right) .
$$

Of course, $H_{0}^{*}, H^{*} \in \mathscr{B}(\mathbf{R}), H_{0}^{*} \subseteq I$ and $m_{1}\left(H_{0}^{*}\right)=m_{1}\left(H^{*}\right)=0$. Moreover, put

$$
S^{*}:=I \times\left(\mathbf{R}^{n}\right)^{k}, \quad \Omega^{*}:=\left(I \backslash H_{0}^{*}\right) \times\left(\mathbf{R} \backslash H^{*}\right)^{n k},
$$

and let $U^{*}=S^{*} \backslash \Omega^{*}$. We observe the following facts. 
(i) $)^{*} \in \mathscr{F}$. Indeed, one has

$$
U^{*}=\left[\left(P_{0}^{-1}\left(H_{0}^{*}\right)\right) \cup\left(\bigcup_{j=1}^{n k} P_{j}^{-1}\left(H^{*}\right)\right)\right] \cap S^{*} .
$$

(ii) $f\left(\Omega^{*}\right) \subseteq \Omega$ (again, we identify $\left(\mathbf{R}^{n}\right)^{k}=\mathbf{R}^{n k}$ in the obvious way).

(iii) $)^{\prime}$ The multifunction $\left.F^{*}\right|_{\Omega^{*}}$ is lower semicontinuous with nonempty closed values. This follows easily from assumption (i), (3.10), (ii)', the continuity of $f$ and $\Omega \subseteq S \backslash U$.

(iv) ${ }^{\prime} F^{*}\left(\Omega^{*}\right) \subseteq \bar{B}_{n}\left(0, r^{*}\right)$, where $r^{*}:=2^{k} T^{k} r$. This follows from (3.10) and (ii)' since

$$
F^{*}\left(\Omega^{*}\right)=2^{k} T^{k} F\left(f\left(\Omega^{*}\right)\right) \subseteq 2^{k} T^{k} F(\Omega) \subseteq 2^{k} T^{k} F(S \backslash U) .
$$

$(\mathrm{v})^{\prime} F^{*}\left(\Omega^{*}\right) \in \mathscr{G}_{n}$. This follows easily from assumption (ii), (3.11) and the definition of $\mathscr{G}_{n}$.

Therefore, taking into account that $\Omega^{*}=S^{*} \backslash U^{*}$, we have that $F^{*}$ satisfies all the assumptions of Theorem 3.1. Consequently, there exists a bounded and upper semicontinuous multifunction $G: \mathbf{R} \times\left(\mathbf{R}^{n}\right)^{k} \rightarrow 2^{\mathbf{R}^{n}}$ with nonempty convex compact values such that

(i) ${ }^{\prime \prime} G\left(\mathbf{R} \times\left(\mathbf{R}^{n}\right)^{k}\right) \subseteq \overline{\operatorname{conv}}\left(F^{*}\left(\Omega^{*}\right)\right) \subseteq \bar{B}_{n}\left(0, r^{*}\right)$;

(ii) $)^{\prime \prime}$ every generalized solution $u \in W^{k, \infty}\left(I, \mathbf{R}^{n}\right)$ of the inclusion

$$
u^{(k)} \in G\left(s, u, u^{\prime}, \ldots, u^{(k-1)}\right)
$$

in $I$ is also a generalized solution of the inclusion $u^{(k)} \in F^{*}\left(s, u, u^{\prime}, \ldots, u^{(k-1)}\right)$ in $I$.

(iii) $^{\prime \prime}$ for every generalized solution $u \in W^{k, \infty}\left(I, \mathbf{R}^{n}\right)$ of the inclusion

$$
u^{(k)} \in G\left(s, u, u^{\prime}, \ldots, u^{(k-1)}\right)
$$

in $I$, one has

$$
\left(s, u(s), u^{\prime}(s), \ldots, u^{(k-1)}(s)\right) \in \Omega^{*} \quad \text { for a.e. } \quad s \in I .
$$

Now, let us define a multifunction $Q: \mathbf{R} \times\left(\mathbf{R}^{n}\right)^{k} \rightarrow 2^{\left(\mathbf{R}^{n}\right)^{k}}$ by putting, for each $(s, \xi)=$ $\left(s, \boldsymbol{\xi}_{0}, \boldsymbol{\xi}_{1}, \ldots, \boldsymbol{\xi}_{k-1}\right) \in \mathbf{R} \times\left(\mathbf{R}^{n}\right)^{k}$,

$$
Q(s, \xi)=Q\left(s, \xi_{0}, \xi_{1}, \ldots, \xi_{k-1}\right)=\left\{\xi_{1}\right\} \times\left\{\xi_{2}\right\} \times \cdots \times\left\{\xi_{k-1}\right\} \times G(s, \xi) .
$$

By [8, Theorem 7.3.14], the multifunction $Q$ is upper semicontinuous.

For every fixed $\xi=\left(\xi_{0}, \xi_{1}, \ldots, \xi_{k-1}\right) \in\left(\mathbf{R}^{n}\right)^{k}$, let us consider the first-order Cauchy problem

$$
\left\{\begin{array}{l}
y^{\prime} \in Q(s, y) \quad \text { in } I \\
y(0)=\xi
\end{array}\right.
$$

Moreover, let

$$
\begin{aligned}
\mathscr{T}_{I}^{Q}(\xi):=\left\{y(s)=\left(y_{0}(s), y_{1}(s), \ldots, y_{k-1}(s)\right) \in\left(W^{1,1}\left(I, \mathbf{R}^{n}\right)\right)^{k}:\right. \\
: y(s) \text { is a generalized solution of (3.12) }\}
\end{aligned}
$$

and

$$
\mathscr{A}_{I}^{Q}(\xi):=\left\{y(1 / 2)=\left(y_{0}(1 / 2), y_{1}(1 / 2), \ldots, y_{k-1}(1 / 2)\right): y \in \mathscr{T}_{I}^{Q}(\xi)\right\}
$$

be the solution set and the attainable set of problem (3.12), respectively. Fix $\xi^{*} \in\left(\mathbf{R}^{n}\right)^{k}$ and choose any $b>0$. Since

$$
\lim _{L \rightarrow+\infty}\left[L^{2}-\left(\left(b+\frac{L}{2}+\left\|\xi^{*}\right\|_{n k}\right)^{2}+\left(r^{*}\right)^{2}\right)\right]=+\infty
$$


there exists $M>0$ such that

$$
\left(b+\frac{M}{2}+\left\|\xi^{*}\right\|_{n k}\right)^{2}+\left(r^{*}\right)^{2}<M^{2} .
$$

We now show that

$$
Q\left(I \times \bar{B}_{n k}\left(\xi^{*}, b+\frac{M}{2}\right)\right) \subseteq \bar{B}_{n k}(0, M) .
$$

To this aim, fix $s \in I$ and $\xi=\left(\xi_{0}, \xi_{1}, \ldots, \xi_{k-1}\right) \in\left(\mathbf{R}^{n}\right)^{k}$ with $\xi \in \bar{B}_{n k}\left(\xi^{*}, b+(1 / 2) M\right)$. Let $\eta=\left(\eta_{0}, \eta_{1}, \ldots, \eta_{k-1}\right) \in Q(s, \xi)$. By the definition of $Q$, there exists $z \in G(s, \xi)$ such that $\eta=\left(\xi_{1}, \xi_{2}, \ldots, \xi_{k-1}, z\right)$. Taking into account that

$$
\|\xi\|_{n k}^{2} \leq\left(b+\frac{M}{2}+\left\|\xi^{*}\right\|_{n k}\right)^{2}
$$

(ii) $^{\prime}$ and (3.13) we have

$$
\begin{aligned}
\|\eta\|_{n k}^{2} & \leq\left\|\xi_{1}\right\|_{n}^{2}+\left\|\xi_{2}\right\|_{n}^{2}+\cdots+\left\|\xi_{k-1}\right\|_{n}^{2}+\|z\|_{n}^{2} \\
& \leq\|\xi\|_{n k}^{2}+\left(r^{*}\right)^{2} \\
& \leq\left(r^{*}\right)^{2}+\left(b+\frac{M}{2}+\left\|\xi^{*}\right\|_{n k}\right)^{2} \\
& <M^{2}
\end{aligned}
$$

Hence, (3.14) is proved. Applying Theorem 2.1 to problem (3.12), we have that, for every $\xi=\left(\xi_{0}, \ldots, \xi_{k-1}\right) \in\left(\mathbf{R}^{n}\right)^{k}$ with $\xi \in B_{n k}\left(\xi^{*}, b\right)$, the solution set $\mathscr{T}_{I}^{Q}(\xi)$ is nonempty. Moreover, the multifunction $\xi \rightarrow \mathscr{T}_{I}^{Q}(\xi)$ is upper semicontinuous from $B_{n k}\left(\xi^{*}, b\right)$ to

$$
W^{1, \infty}\left(I, \mathbf{R}^{n k}\right)=\left(W^{1, \infty}\left(I, \mathbf{R}^{n}\right)\right)^{k}
$$

with nonempty compact connected values (it is quite immediate to check that the topology $\sigma_{n k, 1}^{I}$ coincides with the product topology $\left.\left(\sigma_{n, 1}^{I}\right)^{k}\right)$. Moreover, Theorem 2.1 implies that $\xi \rightarrow \mathscr{A}_{I}^{Q}(\xi)$ is upper semicontinuous in $B_{n k}\left(\xi^{*}, b\right)$ with nonempty compact connected values. Since $\xi^{*} \in$ $\left(\mathbf{R}^{n}\right)^{k}$ is arbitrary, we get that

(i) $^{\prime \prime \prime}$ the solution set $\mathscr{T}_{I}^{Q}(\xi)$ is nonempty for every $\xi \in\left(\mathbf{R}^{n}\right)^{k}$. Moreover, the multifunction $\xi \rightarrow \mathscr{T}_{I}^{Q}(\xi)$ is upper semicontinuous from $\left(\mathbf{R}^{n}\right)^{k}$ to $\left[W^{1, \infty}\left(I, \mathbf{R}^{n}\right)\right]^{k}$ with nonempty compact connected values;

(ii) $)^{\prime \prime \prime}$ the multifunction $\xi \rightarrow \mathscr{A}_{I}^{Q}(\xi)$ is upper semicontinuous from $\left(\mathbf{R}^{n}\right)^{k}$ to $\left(\mathbf{R}^{n}\right)^{k}$ with nonempty compact connected values.

Now, let

$$
P_{1}^{*}:\left[W^{1, \infty}\left(I, \mathbf{R}^{n}\right)\right]^{k} \rightarrow W^{1, \infty}\left(I, \mathbf{R}^{n}\right)
$$

be the first projection. For each $v \in W^{k, \infty}\left(I, \mathbf{R}^{n}\right)$, let $y_{v}: I \rightarrow\left(\mathbf{R}^{n}\right)^{k}$ be defined by putting, for every $s \in I$,

$$
y_{v}(s)=\left(v(s), v^{\prime}(s), \ldots, v^{(k-1)}(s)\right)
$$

and let

$$
E:=\left\{y_{v}: v \in W^{k, \infty}\left(I, \mathbf{R}^{n}\right)\right\} .
$$

By Proposition 2.2, we have that $E$ is a closed subset of $\left[W^{1, \infty}\left(I, \mathbf{R}^{n}\right)\right]^{k}, P_{1}^{*}(E)=W^{k, \infty}\left(I, \mathbf{R}^{n}\right)$, and the function

$$
\left.P_{1}^{*}\right|_{E}:\left(E,\left(\sigma_{n, 1}^{I}\right)^{k}\right) \rightarrow\left(W^{k, \infty}\left(I, \mathbf{R}^{n}\right), \sigma_{n, k}^{I}\right)
$$


is continuous. For each $\xi=\left(\xi_{0}, \xi_{1}, \ldots, \xi_{k-1}\right) \in\left(\mathbf{R}^{n}\right)^{k}$, let us consider the Cauchy problem

$$
\left\{\begin{array}{l}
u^{(k)} \in G\left(t, u, u^{\prime}, \ldots, u^{(k-1)}\right) \quad \text { in } I, \\
u^{(i)}(0)=\xi_{i}
\end{array}\right.
$$

and let

$$
\mathscr{T}_{I}^{G}(\xi):=\left\{u \in W^{k, 1}\left(I, \mathbf{R}^{n}\right): u \text { is a generalized solution of (3.15) }\right\}
$$

its solution set. Since $G$ is bounded, we have $\mathscr{T}_{I}^{G}(\xi) \subseteq W^{k, \infty}\left(I, \mathbf{R}^{n}\right)$ for all $\xi \in\left(\mathbf{R}^{n}\right)^{k}$.

Fix $\xi=\left(\xi_{0}, \xi_{1}, \ldots, \xi_{k-1}\right) \in\left(\mathbf{R}^{n}\right)^{k}$, and let

$$
w(s)=\left(w_{0}(s), w_{1}(s), \ldots, w_{k-1}(s)\right) \in \mathscr{T}_{I}^{Q}(\xi) \subseteq\left[W^{1, \infty}\left(I, \mathbf{R}^{n}\right)\right]^{k} .
$$

Hence, $w^{\prime}(s) \in Q(s, w(s))$ for a.e. $s \in I$. Therefore, by the definition of $Q$, we have, for a.e. $s \in I$,

$$
\begin{gathered}
w_{1}(s)=w_{0}^{\prime}(s), \quad w_{2}(s)=w_{1}^{\prime}(s), \quad \ldots, \quad w_{k-1}(s)=w_{k-2}^{\prime}(s), \\
w_{k-1}^{\prime}(s) \in G\left(s, w_{0}(s), \ldots, w_{k-1}(s)\right) .
\end{gathered}
$$

Since the functions $w_{0}, w_{1}, \ldots, w_{k-1}$ are absolutely continuous, by the same standard argument as in the proof of Proposition 2.2, it follows that $w_{0} \in C^{k-1}\left(I, \mathbf{R}^{n}\right)$. For every $s \in I$, one has

$$
w_{1}(s)=w_{0}^{\prime}(s), \quad w_{2}(s)=w_{0}^{\prime \prime}(s), \quad \ldots \quad w_{k-1}(s)=w_{0}^{(k-1)}(s) .
$$

Hence, $\left.w_{0} \in W^{k, \infty}\left(I, \mathbf{R}^{n}\right)\right)$ and

$$
w_{0}^{(k)}(s)=w_{k-1}^{\prime}(s) \in G\left(s, w_{0}(s), \ldots, w_{0}^{(k-1)}(s)\right)
$$

for a.e. $s \in I$. Moreover, one has

$$
w_{0}^{(j)}(0)=w_{j}(0)=\xi_{j} \quad \text { for every } j=0, \ldots, k-1 .
$$

Hence, $w_{0} \in \mathscr{T}_{I}^{G}(\xi)$ and $w=y_{w_{0}} \in E$. Therefore, we obtain that, for every $\xi=\left(\xi_{0}, \ldots, \xi_{k-1}\right) \in$ $\left(\mathbf{R}^{n}\right)^{k}$

$$
\mathscr{T}_{I}^{Q}(\xi) \subseteq E, \quad \text { and } \quad P_{1}^{*}\left(\mathscr{T}_{I}^{Q}(\xi)\right) \subseteq \mathscr{T}_{I}^{G}(\xi) .
$$

Now, let us define a multifunction $\mathbb{I}:\left(\mathbf{R}^{n}\right)^{k} \rightarrow 2^{W^{k, \infty}\left(I, \mathbf{R}^{n}\right)}$ by putting, for each $\xi=\left(\xi_{0}, \xi_{1}, \ldots, \xi_{k-1}\right)$ $\in\left(\mathbf{R}^{n}\right)^{k}$,

$$
\mathbb{I}(\xi):=P_{1}^{*}\left(\mathscr{T}_{I}^{Q}(\xi)\right)
$$

By (3.16), we get that

$$
\mathbb{I}(\xi) \subseteq \mathscr{T}_{I}^{G}(\xi) \quad \text { for every } \quad \xi=\left(\xi_{0}, \xi_{1}, \ldots, \xi_{k-1}\right) \in\left(\mathbf{R}^{n}\right)^{k} .
$$

By (i) $)^{\prime \prime \prime},(3.16)$ and the continuity of the function

$$
\left.P_{1}^{*}\right|_{E}:\left(E,\left(\sigma_{n, 1}^{I}\right)^{k}\right) \rightarrow\left(W^{k, \infty}\left(I, \mathbf{R}^{n}\right), \sigma_{n, k}^{I}\right),
$$

we get that

(a) $)^{\prime}$ the multifunction $I I$ is upper semicontinuous (with respect to the topology $\sigma_{n, k}^{I}$ of the space $W^{k, \infty}\left(I, \mathbf{R}^{n}\right)$ ), with nonempty, compact and connected values.

Now, if we denote by $\Pi_{1}:\left(\mathbf{R}^{n}\right)^{k} \rightarrow \mathbf{R}^{n}$ the first projection from $\left(\mathbf{R}^{n}\right)^{k}$ to $\mathbf{R}^{n}$, it is easy to see from the above construction that, for every $\xi=\left(\xi_{0}, \ldots, \xi_{k-1}\right) \in\left(\mathbf{R}^{n}\right)^{k}$,

$$
\{u(1 / 2): u \in \mathbb{I}(\xi))\}=\Pi_{1}\left(\mathscr{A}_{I}^{Q}(\xi)\right) .
$$

Thus, taking into account (ii) $)^{\prime \prime \prime}$ and the continuity of $\Pi_{1}$, we have that 
(b) ${ }^{\prime}$ the multifunction

$$
\left.R: \xi \in\left(\mathbf{R}^{n}\right)^{k} \rightarrow R(\xi):=\{u(1 / 2): u \in \mathbb{I}(\xi))\right\}
$$

is upper semicontinuous in $\left(\mathbf{R}^{n}\right)^{k}$ with nonempty, compact and connected values.

In order to conclude the proof, let $h:\left(\mathbf{R}^{n}\right)^{k} \rightarrow\left(\mathbf{R}^{n}\right)^{k}$ be the continuous functions defined by putting, for each $\xi=\left(\xi_{0}, \ldots, \xi_{k-1}\right) \in\left(\mathbf{R}^{n}\right)^{k}$,

$$
h(\xi)=\left(\xi_{0}, 2 T \xi_{1}, 2^{2} T^{2} \xi_{2}, \ldots, 2^{k-1} T^{k-1} \xi_{k-1}\right) .
$$

Moreover, let

$$
\phi:\left(W^{k, \infty}\left(I, \mathbf{R}^{n}\right), \sigma_{n, k}^{I}\right) \rightarrow\left(W^{k, \infty}\left([0, T], \mathbf{R}^{n}\right), \sigma_{n, k}^{[0, T]}\right)
$$

be defined by putting, for each $u \in W^{k, \infty}\left(I, \mathbf{R}^{n}\right)$,

$$
\phi(u)(t)=u\left(\frac{1}{2 T} t\right) \quad \text { for every } t \in[0, T] .
$$

Arguing as above, it is not difficult to check that $\phi$ is continuous. Moreover, one has

$$
\phi\left(\mathscr{T}_{I}^{G}(h(\xi))\right) \subseteq \mathscr{T}_{[0, T]}^{F}(\xi) \quad \text { for every } \quad \xi=\left(\xi_{0}, \xi_{1}, \ldots, \xi_{k-1}\right) \in\left(\mathbf{R}^{n}\right)^{k} .
$$

To see this, fix $\xi=\left(\xi_{0}, \xi_{1}, \ldots, \xi_{k-1}\right) \in\left(\mathbf{R}^{n}\right)^{k}$ and a function $v \in \phi\left(\mathscr{T}_{I}^{G}(h(\xi))\right)$. Therefore, there exists $u \in \mathscr{T}_{I}^{G}(h(\xi))$ such that $v=\phi(u)$. By the definition of $\phi(u)$, for every $j=0, \ldots, k-1$, we have

$$
v^{(j)}(t)=\frac{1}{2^{j} T^{j}} u^{(j)}\left(\frac{t}{2 T}\right) \text { for all } t \in[0, T] .
$$

Moreover, taking into account (ii) $)^{\prime \prime}$, there exists a set $K \subseteq I$ with $m_{1}(K)=0$ such that

$$
v^{(k)}(t)=\frac{1}{2^{k} T^{k}} u^{(k)}\left(\frac{t}{2 T}\right) \text { for all } t \in[0, T] \backslash(2 T K)
$$

and

$$
u^{(k)}(s) \in F^{*}\left(s, u(s), u^{\prime}(s), \ldots, u^{(k-1)}(s)\right) \quad \text { for all } \quad s \in I \backslash K .
$$

Consequently, for every $j=0, \ldots, k-1$, we have

$$
v^{(j)}(0)=\frac{1}{2^{j} T^{j}} u^{(0)}=\xi_{j}
$$

Moreover, for every $t \in[0, T] \backslash(2 T K)$, taking into account (3.21), one has

$$
\begin{aligned}
v^{(k)}(t) & =\frac{1}{2^{k} T^{k}} u^{(k)}\left(\frac{t}{2 T}\right) \\
& \in \frac{1}{2^{k} T^{k}} F^{*}\left(\frac{t}{2 T}, u\left(\frac{t}{2 T}\right), u^{\prime}\left(\frac{t}{2 T}\right), \ldots, u^{(k-1)}\left(\frac{t}{2 T}\right)\right) \\
& =\frac{1}{2^{k} T^{k}} F^{*}\left(\frac{t}{2 T}, v(t), 2 T v^{\prime}(t), \ldots, 2^{k-1} T^{k-1} v^{(k-1)}(t)\right) \\
& =F\left(t, v(t), v^{\prime}(t), \ldots, v^{(k-1)}(t)\right) .
\end{aligned}
$$

Hence, $v \in \mathscr{T}_{[0, T]}^{F}(\xi)$. 
Now, let $\Phi:\left(\mathbf{R}^{n}\right)^{k} \rightarrow 2^{W^{k, \infty}\left([0, T], \mathbf{R}^{n}\right)}$ be the multifunction defined by setting, for each $\xi \in$ $\left(\mathbf{R}^{n}\right)^{k}$

$$
\Phi(\xi)=\phi(\mathbb{I}(h(\xi)))
$$

By (a) ${ }^{\prime}$ and the continuity of $\phi$ and $h$, it follows that $\Phi$ is upper semicontinuous (with respect to the topology $\sigma_{n, k}^{[0, T]}$ of $\left.W^{k, \infty}\left([0, T], \mathbf{R}^{n}\right)\right)$ with nonempty, compact and connected values. Moreover, by (3.17) and (3.18), we have that $\Phi(\xi) \subseteq \mathscr{T}_{[0, T]}^{F}(\xi)$ for every $\xi \in\left(\mathbf{R}^{n}\right)^{k}$.

In order to prove conclusion (c), it is immediate to check that, for every $\xi \in\left(\mathbf{R}^{n}\right)^{k}$,

$$
\{v(T): v \in \Phi(\xi)\}=\{u(1 / 2): u \in \mathbb{I}(h(\xi))\} .
$$

Consequently, conclusion (c) follows from the continuity of $h$ and (b)'.

Now, in order to prove conclusion (d), we fix a point $\xi \in\left(\mathbf{R}^{n}\right)^{k}$ and $v \in \Phi(\xi)$. By the definition of $\Phi$, there exists $u \in \mathbb{I}(h(\xi))$ such that $v=\phi(u)$. By (3.17), in particular, we have $u \in \mathscr{T}_{I}^{G}(h(\xi))$. Hence, by (iii) ${ }^{\prime \prime}$, there exists a set $K_{1} \subseteq I$ with $m_{1}\left(K_{1}\right)=0$ such that

$$
\left(s, u(s), u^{\prime}(s), \ldots, u^{(k-1)}(s)\right) \in \Omega^{*} \quad \text { for every } \quad s \in I \backslash K_{1} .
$$

Fix $t \in[0, T] \backslash\left(2 T K_{1}\right)$. Putting $s=(1 / 2 T) t \in I \backslash K_{1}$, and using (3.19) and (3.9), we have

$$
\begin{aligned}
\left(t, v(t), v^{\prime}(t) ; \ldots, v^{(k-1)}(t)\right) & =\left(t, u\left(\frac{t}{2 T}\right), \frac{1}{2 T} u^{\prime}\left(\frac{t}{2 T}\right), \ldots, \frac{1}{2^{k-1} T^{k-1}} u^{(k-1)}\left(\frac{t}{2 T}\right)\right) \\
& =\left(2 T s, u(s), \frac{1}{2 T} u^{\prime}(s), \ldots, \frac{1}{2^{k-1} T^{k-1}} u^{(k-1)}(s)\right) \\
& =f\left(s, u(s), u^{\prime}(s), \ldots, u^{(k-1)}(s)\right) .
\end{aligned}
$$

By (3.22), (ii)' and (3.8) we immediately get

$$
\left(t, v(t), v^{\prime}(t) ; \ldots, v^{(k-1)}(t)\right) \in \Omega \subseteq(S \backslash U),
$$

as desired. Therefore, conclusion (d) is proved since $m_{1}\left(2 T K_{1}\right)=0$.

Finally, in order to prove conclusion (e), we observe that, by the definition of the topology $\sigma_{n, k}^{[0, T]}$, the function

$$
v \in W^{k, \infty}\left([0, T], \mathbf{R}^{n}\right) \rightarrow v^{(k)} \in L^{\infty}\left([0, T], \mathbf{R}^{n}\right)
$$

is continuous (where, as before, $L^{\infty}\left([0, T], \mathbf{R}^{n}\right)$ is considered with its weak-star topology). Hence, conclusion (e) follows at once from conclusion (b). The proof is now complete.

Remark 3.1. The assumptions on the multifuncion $F$ in Theorems 3.1 and 3.2 do not imply any kind of semicontinuity for $F$. To see this, fix $T>0$, and consider the case where $n=k=1$, and $F:[0, T] \times \mathbf{R} \rightarrow 2^{\mathbf{R}}$ is defined by putting, for each $(t, x) \in[0, T] \times \mathbf{R}$,

$$
F(t, x)= \begin{cases}{\left[-2 x^{2},-x^{2}\right],} & t \in[0, T], x \in \mathbf{Q}, \\ {[2+\sin x, 4]} & t \in[0, T], x \in \mathbf{R} \backslash \mathbf{Q} .\end{cases}
$$

It is immediate to check that all the assumptions of Theorems 3.1 and 3.2 are satisfied by taking $U=[0, T] \times \mathbf{Q}$ (in particular, the lower semicontinuity of $\left.F\right|_{S \backslash U}$ follows by [8, Theorems 7.3.8 and 7.3.17]). However, for every fixed $(t, x) \in[0, T] \times \mathbf{R}$, such a multifunction $F$ is neither lower nor upper semicontinuous at $(t, x)$. 
Remark 3.2. Theorems 3.1 and 3.2 do not hold without the assumption $F(S \backslash U) \in \mathscr{G}_{n}$. To see this, fix $T>0$, and consider the function $f:[0, T] \times \mathbf{R} \rightarrow \mathbf{R}$ defined by setting

$$
f(t, x)= \begin{cases}0, & t \in[0, T], x \neq 0, \\ 1, & t \in[0, T], x=0 .\end{cases}
$$

As showed in [11, Example 1], for such a function $f$ the Cauchy problem

$$
\left\{\begin{array}{l}
u^{\prime}=f(t, u) \text { in }[0, T] \\
u(0)=0
\end{array}\right.
$$

has no generalized solutions in $[0, T]$. Now, we consider the multifunction $F:[0, T] \times \mathbf{R} \rightarrow 2^{\mathbf{R}}$ defined by setting, for each $(t, x) \in[0, T] \times \mathbf{R}$,

$$
F(t, x)=\{f(t, x)\} .
$$

It is immediate to check that such a multifunction $F$ satisfies all the assumptions of Theorems 3.1 and 3.2 (with $n=k=1, U=[0, T] \times\{0\}$ ), except the assumption $F(S \backslash U) \in \mathscr{G}_{1}$. Now, observe that the conclusion of Theorem 3.2 does not hold since $\mathscr{T}_{[0, T]}^{F}(0)=$. Moreover, even the conclusion of Theorem 3.1 is not true for such a multifunction $F$. To see this, we assume that there exists a multifunction $G: \mathbf{R} \times \mathbf{R} \rightarrow 2^{\mathbf{R}}$ satisfying the conclusion of Theorem 3.1. Then, by Theorem 2.1, there should exist an absolutely continuous $\bar{u}:[0, T] \rightarrow \mathbf{R}$ such that $\bar{u}(0)=0$ and $\bar{u}^{\prime}(t) \in G(t, \bar{u}(t))$ for a.e. $t \in[0, T]$. Consequently, $\bar{u}^{\prime}(t) \in F(t, \bar{u}(t))=\{f(t, \bar{u}(t))\}$ for a.e. $t \in[0, T]$, which is a contradiction since the Cauchy problem (3.24) has no generalized solutions.

\section{An Application to Ordinary DifFEREnTial Equations}

Let $n, k \in \mathbf{N}$, and $T>0$. Let $Y \subseteq \mathbf{R}^{n}$ be a nonempty set. Let $g: Y \rightarrow \mathbf{R}$ and $f:[0, T] \times\left(\mathbf{R}^{n}\right)^{k} \rightarrow$ $\mathbf{R}$ be two given functions, and let $\xi=\left(\xi_{0}, \xi_{1}, \ldots, \xi_{k-1}\right) \in\left(\mathbf{R}^{n}\right)^{k}$. We recall that a generalized solution of the Cauchy problem

$$
\begin{cases}g\left(u^{(k)}\right)=f\left(t, u, u^{\prime}, \ldots, u^{(k-1)}\right) & \text { in }[0, T], \\ u^{(i)}(0)=\xi_{i}, & i=0,1, \ldots, k-1,\end{cases}
$$

is a function $u \in W^{k, 1}\left([0, T], \mathbf{R}^{n}\right)$ such that

$$
u^{(k)}(t) \in Y \quad \text { and } \quad g\left(u^{(k)}(t)\right)=f\left(t, u(t), u^{\prime}(t), \ldots, u^{(k-1)}(t)\right) \quad \text { for a.e. } t \in[0, T],
$$

and $u^{(i)}(0)=\xi_{i}$ for every $i=0,1, \ldots, k-1$. For each fixed $\xi=\left(\xi_{0}, \xi_{1}, \ldots, \xi_{k-1}\right) \in\left(\mathbf{R}^{n}\right)^{k}$, we denote by

$$
\mathscr{S}(\xi)=\left\{u \in W^{k, 1}\left([0, T], \mathbf{R}^{n}\right): u \text { is a generalized solution of (4.1) }\right\}
$$

the solution set of problem (4.1). Of course, if $Y$ is bounded, then every generalized solution of problem (4.1) belongs to $W^{k, \infty}\left([0, T], \mathbf{R}^{n}\right)$.

Our aim in this section is to apply Theorem 3.2 to obtain an existence and qualitative result for the generalized solutions of the implicit problem (4.1) associated with a discontinuous function $f$. As far as we know, there are not many results in this direction, even for the special case $k=1$. In this latter case, some existence and qualitative results can be found in [2] and [11]. 
In the explicit case where $k=1$ and $g\left(u^{\prime}\right)=u^{\prime}$, conversely, the Cauchy problem (4.1) has been widely studied in the past (see, for instance, $[12,13,14,15,16,17,18]$ ).

The following is our result.

Theorem 4.1. Let $n, k \in \mathbf{N}$, and $T>0$. Let $Y \in \mathscr{G}_{n}$ be a nonempty, compact, connected and locally connected subset of $\mathbf{R}^{n}$. Let $g: Y \rightarrow \mathbf{R}$ and $f:[0, T] \times\left(\mathbf{R}^{n}\right)^{k} \rightarrow \mathbf{R}$ be two given functions with $g$ continuous. Put $S:=[0, T] \times\left(\mathbf{R}^{n}\right)^{k}$. Assume that there exists a set $U \subseteq S$ with $U \in \mathscr{F}$ such that

(i) $\left.f\right|_{S \backslash U}$ is continuous;

(ii) $f(S \backslash U) \subseteq g(Y)$;

(iii) for every $r \in] \inf g(Y), \sup g(Y)\left[\right.$, int $_{Y}\left(g^{-1}(r)\right)=\emptyset$.

Then, for every $\xi=\left(\xi_{0}, \xi_{1}, \ldots, \xi_{k-1}\right) \in\left(\mathbf{R}^{n}\right)^{k}$, the solution set $\mathscr{S}(\xi)$ of problem (4.1) is nonempty. Moreover, there exists a multifunction

$$
\Phi:\left(\mathbf{R}^{n}\right)^{k} \rightarrow 2^{W^{k, \infty}\left([0, T] ; \mathbf{R}^{n}\right)}
$$

such that

(a) $\Phi(\xi) \subseteq \mathscr{S}(\xi)$ for all $\xi \in\left(\mathbf{R}^{n}\right)^{k}$;

(b) $\Phi$ is upper semicontinuous (with respect to the topology $\sigma_{n, k}^{[0, T]}$ of $W^{k, \infty}\left([0, T] ; \mathbf{R}^{n}\right)$ ) with nonempty, compact and connected values;

(c) the multifunction

$$
\xi \in\left(\mathbf{R}^{n}\right)^{k} \rightarrow\{u(T): u \in \Phi(\xi)\}
$$

is upper semicontinuous with nonempty connected and compact values;

(d) for every $\xi \in\left(\mathbf{R}^{n}\right)^{k}$ and every $u \in \Phi(\xi)$, one has

$$
\left(t, u(t), u^{\prime}(t), \ldots, u^{(k-1)}(t)\right) \in S \backslash U \quad \text { for a.e. } \quad t \in[0, T]
$$

(e) the multifunction

$$
\xi \in\left(\mathbf{R}^{n}\right)^{k} \rightarrow\left\{u^{(k)} \in L^{\infty}\left([0, T], \mathbf{R}^{n}\right): u \in \Phi(\xi)\right\}
$$

is upper semicontinuous (with compact connected values) with respect to the weak-star topology of $L^{\infty}\left([0, T], \mathbf{R}^{n}\right)$.

Proof. By [19, Theorem 2.4], the function $g$ is inductively open on $Y$. That is, there exists a set $Y^{*} \subseteq Y$ such that $g\left(Y^{*}\right)=g(Y)$ and the function $\left.g\right|_{Y^{*}}: Y^{*} \rightarrow g(Y)$ is open. Let $T: S \backslash U \rightarrow 2^{Y}$ be the multifunction defined by setting, for each $(t, \xi)=\left(t, \xi_{0}, \xi_{1}, \ldots, \xi_{k-1}\right) \in S \backslash U$,

$$
T\left(t, \xi_{0}, \xi_{1}, \ldots, \xi_{k-1}\right)=\overline{g^{-1}\left(f\left(t, \xi_{0}, \xi_{1}, \ldots, \xi_{k-1}\right)\right) \cap Y^{*}} .
$$

By the above construction, and assumptions (i) and (ii) and [8, Proposition 7.3.3], it is not difficult to check that $T$ is lower semicontinuous with nonempty closed values. Moreover, $T(S \backslash U) \subseteq Y$ is bounded by the compactness of $Y$. Choose any point $y^{*} \in T(S \backslash U)$, and let $F: S \rightarrow Y$ be the multifunction defined by putting, for each $(t, \xi)=\left(t, \xi_{0}, \xi_{1}, \ldots, \xi_{k-1}\right) \in S$,

$$
F\left(t, \xi_{0}, \xi_{1}, \ldots, \xi_{k-1}\right)= \begin{cases}T\left(t, \xi_{0}, \xi_{1}, \ldots, \xi_{k-1}\right) & \text { if }\left(t, \xi_{0}, \xi_{1}, \ldots, \xi_{k-1}\right) \in S \backslash U \\ \left\{y^{*}\right\} & \text { if }\left(t, \xi_{0}, \xi_{1}, \ldots, \xi_{k-1}\right) \in U\end{cases}
$$

Since $F(S \backslash U)=T(S \backslash U) \subseteq Y$, the multifunction $F$ satisfies all the assumptions of Theorem 3.2. Consequently, there exists a multifunction $\Phi:\left(\mathbf{R}^{n}\right)^{k} \rightarrow 2^{W^{k, \infty}\left([0, T], \mathbf{R}^{n}\right)}$ satisfying the conclusion of Theorem 3.2. 
Now, we claim that $\Phi(\xi) \subseteq \mathscr{S}(\xi)$ for every $\xi \in\left(\mathbf{R}^{n}\right)^{k}$. To this aim, fix $\xi \in\left(\mathbf{R}^{n}\right)^{k}$ and $u \in \Phi(\xi)$. By the properties (a) and (d) described in the conclusion of Theorem 3.2, there exists a set $K \subseteq[0, T]$ with $m_{1}(K)=0$ such that

$$
u^{(k)}(t) \in F\left(t, u(t), u^{\prime}(t), \ldots, u^{(k-1)}(t)\right) \quad \text { and } \quad\left(t, u(t), u^{\prime}(t), \ldots, u^{(k-1)}(t)\right) \in S \backslash U
$$

for all $t \in[0, T] \backslash K$. Hence,

$$
u^{(k)}(t) \in Y \quad \text { and } \quad u^{(k)}(t) \in T\left(t, u(t), u^{\prime}(t), \ldots, u^{(k-1)}(t)\right) \quad \text { for all } t \in[0, T] \backslash K .
$$

Consequently, for every $t \in[0, T] \backslash K$, taking into account the definition of $T$, the continuity of $g$ and the closedness of $Y$, we get

$$
\begin{aligned}
u^{(k)}(t) & \in \overline{g^{-1}\left(f\left(t, u(t), u^{\prime}(t), \ldots, u^{(k-1)}(t)\right)\right) \cap Y^{*}} \\
& \subseteq \overline{g^{-1}\left(f\left(t, u(t), u^{\prime}(t), \ldots, u^{(k-1)}(t)\right)\right)} \\
& =g^{-1}\left(f\left(t, u(t), u^{\prime}(t), \ldots, u^{(k-1)}(t)\right)\right) .
\end{aligned}
$$

Hence, we get

$$
g\left(u^{(k)}(t)\right)=f\left(t, u(t), u^{\prime}(t), \ldots, u^{(k-1)}(t)\right), \quad \forall t \in[0, T] \backslash K,
$$

hence $u \in \mathscr{S}(\xi)$, as claimed. At this point, our conclusion follows at once by the conclusion of Theorem 3.2.

Remark 4.1. The assumptions of Theorem 4.1 do not imply the continuity of the function $f:[0, T] \times\left(\mathbf{R}^{n}\right)^{k} \rightarrow \mathbf{R}$ with respect to the second variable. To see this, fix $T>0$, and consider the case $n=k=1, Y=[2,3], g(y)=y$, and $f:[0, T] \times \mathbf{R} \rightarrow \mathbf{R}$ defined by putting, for each $(t, x) \in[0, T] \times \mathbf{R}$,

$$
f(t, x)= \begin{cases}1, & t \in[0, T], x \in \mathbf{Q}, \\ 2 & t \in[0, T], x \in \mathbf{R} \backslash \mathbf{Q} .\end{cases}
$$

It is immediate to check that all the assumptions of Theorems 4.1 are satisfied by taking $U=$ $[0, T] \times \mathbf{Q}$. However, for every $t \in[0, T]$, function $f(t, \cdot)$ is discontinuous at each point $x \in \mathbf{R}$.

Indeed, in Theorem 4.1 we make requirements only on $\left.f\right|_{S \backslash U}$. Consequently, $f$ could even be defined only over $S \backslash U$. This fact is the main peculiarity of Theorem 4.1 since in the literature (up to our knowledge) the function $f(t, \cdot)$ is usually defined either on the whole space $\left(\mathbf{R}^{n}\right)^{k}$, or on a closed set with empty interior, or on a ball (see, for instance, [20, 21, 22, 23, 24, 25, 26, 27] and the references therein).

Remark 4.2. Theorems 4.1 does not hold without the assumption $Y \in \mathscr{G}_{n}$. To see this, let $T>0, n=k=1, Y=[0,1], g(y)=y, U=[0, T] \times\{0\}$, and let $f:[0, T] \times \mathbf{R} \rightarrow \mathbf{R}$ be defined by (3.23). It is immediate to check that all the assumptions of Theorem 4.1 are satisfied except for the assumption $Y \in \mathscr{G}_{1}$. However, the conclusion of Theorem 4.1 does not hold since we have $\mathscr{S}(0)=$ in this case (see Remark 3.2).

\section{Acknowledgement}

The author is grateful to the referees for their useful comments, which improved this paper a lot. 


\section{REFERENCES}

[1] A. Bressan, Upper and lower semicontinuous differential inclusions: a unified approach, In: H.J. Sussmann, (ed.) Nonlinear Controllability and Optimal Control, Marcel Dekker, New York, 1990.

[2] P. Cubiotti, J.C. Yao, On the Cauchy problem for a class of differential inclusions with applications, Appl. Anal. 99 (2020), 2543-2554.

[3] P. Cubiotti, On the Cauchy problem for implicit differential equations with discontinuous right-hand side, J. Nonlinear Convex Anal. 21 (2020), 1027-1033.

[4] P. Cubiotti, J.C. Yao, Two-point problem for vector differential inclusions with discontinuous right-hand side, Appl. Anal. 93 (2014), 1811-1823.

[5] P. Cubiotti, J.C. Yao, On the two-point problem for implicit second-order ordinary differential equations, Boundary Value Probl. 2015 (2015), 211.

[6] J.P. Aubin, A. Cellina, Differential Inclusions, Springer-Verlag, Berlin, 1984.

[7] J.P. Aubin, H. Frankowska, Set-Valued Analysis, Birkhäuser, Boston, 1990.

[8] E. Klein, A.C. Thompson, Theory of Correspondences, John Wiley and Sons, New York, 1984.

[9] A. Villani, On Lusin's condition for the inverse function, Rend. Circ. Mat. Palermo 33 (1984), 331-335.

[10] E. Hewitt, K. Stromberg, Real and Abstract Analysis, Springer-Verlag, Berlin, 1965.

[11] B. Ricceri, Lipschitzian solutions of the implicit Cauchy problem $g\left(x^{\prime}\right)=f(t, x), x(0)=0$, with $f$ discontinuous in $x$, Rend. Circ. Mat. Palermo Serie II 34 (1985), 127-135.

[12] P. Binding, The differential equation $\dot{x}=f \circ x$, J. Differential Equations 31 (1979), 183-199.

[13] A. Cabini, S. Querci, Equazioni differenziali del primo ordine con secondo membro discontinuo rispetto all'incognita, Rend. Ist. Mat. Univ. Trieste 1 (1969), 89-97.

[14] A.F. Filippov, Differential equations with discontinuous right-hand side, Amer. Math. Soc. Transl. 42 (1964), 199-231.

[15] S. Giuntini, G. Pianigiani, Equazioni differenziali ordinarie con secondo membro discontinuo, Atti Sem. Mat. Fis. Univ. Modena 23 (1974), 233-240.

[16] V.M. Matrosov, On differential equations and inequalities with discontinuous right-hand sides I, Differential Equations 3 (1967), 395-409.

[17] A. Pucci, Sistemi di equazioni differenziali con secondo membro discontinuo rispetto all'incognita, Rend. Ist. Mat. Univ. Trieste 3 (1971), 75-80.

[18] R. Sentis, Equations différentielles á second membre measurable, Boll. Un. Mat. Ital. 15-B (1978), 724-742.

[19] B. Ricceri, Sur la semi-continuité inférieure de certaines multifonctions, C.R. Acad. Sc. Paris 294 (1982), 265-267.

[20] S. Carl, S. Heikkilä, On discontinuous implicit evolution equations, J. Math. Anal. Appl. 219 (1998), 455471.

[21] J. Ángel Cid, On uniqueness criteria for systems of ordinary differential equations, J. Math. Anal. Appl. 281 (2003), 264-275.

[22] J. Ángel Cid, S. Heikkilä, R.L. Pouso, Uniqueness and existence results for ordinary differential equations, J. Math. Anal. Appl. 316 (2006), 178-188.

[23] S. Heikkilä, M. Kumpulainen, S. Seikkala, Existence, uniqueness and comparison results for a differential equation with discontinuous nonlinearities, J. Math. Anal. Appl. 201 (1996), 478-488.

[24] S. Heikkilä, V. Lakshmikantham, A unified theory for first-order discontinuous scalar differential equations, Nonlinear Anal. 26 (1996), 785-797.

[25] R.L. Pouso, On the Cauchy problem for first order discontinuous ordinary differential equations, J. Math. Anal. Appl. 264 (2001), 230-252.

[26] B. Ricceri, On the Cauchy problem for the differential equation $f\left(t, x, x^{\prime}, \ldots, x^{(k)}\right)=0$, Glasgow Math. J. 33 (1991), 343-348.

[27] J.R.L. Webb, S.C. Welsh, Existence and uniqueness of initial value problems for a class of second-order differential equations, J. Differential Equations 82 (1989), 314-321. 